\title{
Genetic Algorithm based Approach to Enhance Network Performance in Multi-rate WLANs
}

\author{
Qiang Ma, Abdullah Al-Dhelaan and Mznah Al-Rodhaan \\ Department of Computer Science, College of Computer \& Information Sciences \\ King Saud University, Riyadh, Saudi Arabia \\ qiang.ma@student.ksu.edu.sa;\{dhelaan,rodhaan@ksu.edu.sa\}
}

\begin{abstract}
In a multi-rate 802.11 WLAN environment, the users' fairness and network throughput is a trade-off problem. Although there are many valuable research papers related to this optimization problem, up to date, none of those researches could offer a rational, clearly designed mathematical model which can be easily and widely implemented using the well known Al algorithms. Thus our research aims to fill such gap. In this paper we define the problem as an informed search NP-hard problem in a practical scenario, and then we will propose a new intuitive simplified mathematical model called Simplified Coefficient of Variation (SCV), by using Genetic Algorithm to implement the SCV model, through controlling the power of Access Points to optimize and enhance the performance of the network. The simulation gives excellent results that indicate our model is efficient and superior to existing method. After the experiment analysis, we use software SAS to further reveal the relationships of three indicators to illustrate the essence of our algorithm and an existing algorithm.
\end{abstract}

Keywords: power control; SCV; genetic algorithm; optimization; cost function; coefficient of variation.

\section{Introduction}

The rapid development of the Internet and the progress of wireless technology are making wireless networks play an increasingly important role in many areas. This is particularly true for the IEEE 802.11 wireless local area network (WLAN) technology. With its development, the increasing demands of service quality and a sharp rise in the number of user groups, the problem has become heavily concentrated in some places such as offices, meeting rooms and other crowded places. In this case, many access points may be allocated, but without an overall channel or power planning and this will result in a large amount of co-channel interference, load imbalance, and network throughput decline, which will degrade the user experience. As it is one of the hot spots in the wireless area, research institutions, academic institutions, and commercial companies have developed many valuable solutions to solve the problems of WLAN, but those solutions cannot be applied easily.

Currently, most research on WLAN technology is mainly focused on the following two aspects:

a) Wireless channel planning. Through different methods, the limited channel resources will be reasonably assigned to all access points (APs) to make it possible to reduce co-channel interference and network overhead in order to improve overall network throughput. 
b) Power control to achieve load balancing. Power control mainly uses the proportional relationship of AP signal strength and the power of the AP selected by the user accessing the wireless network, increasing or decreasing the power to adjust the signal strength of the AP. It thus changes the access topology of the user-AP in the network in order to reduce the scheduling overhead, improve load balance etc.

This article considers both aspects above. The rest of the paper is organized as follows: related work is discussed in Section 2. Section 3 shows the motivation. As background knowledge, a brief introduction of genetic algorithm will be given in Section 4, and then we start to explain our new model SCV and apply the Genetic Algorithm. The simulation modeling using Matlab is explained in Section 5. After this, we analyze the results using the statistics software SAS in Section 6 and finally give conclusions in Section 7.

\section{Related Work}

According to IEEE 802.11, a high-density WLAN deployment environment offers a short distance between APs and users. In this case, each user will connect with the AP by the strongest received signal strength indicator (RSSI) by default. We know that the users are not uniformly distributed in an area, which makes some APs connect more users than the other APs. This will produce the load imbalance problem, as some APs are hungry while some APs are overloaded. This situation results in unfair use of resources.

As a part of our research, the basic solution has been introduced in [1]. In order to improve the Quality of Service, the authors in [2] provided an enhanced method called DCF which providing weighted fairness among multiple priority classes in 802.11-based WLAN to properly control the transmission probability of nodes. The method was expected to achieve not only the weighted fairness but also maximize the system throughput and minimize the frame delay at the same time.

The authors in [3] proposed an Improved Power Control MAC (IPCM) protocol which improves the throughput and yields energy saving. The protocol adopted optimal transmission power to send all kinds of packets in order to save the energy, which also made spatial reuse of the wireless channels, and achieved the maximum throughput compared to the other schemes.

The authors in [4] introduced three strategies which were Dirty Paper Coding (DPC) strategy, Noise strategy, and Opportunistic Interference Cancellation (OIC) strategy. Then they compared the achievable rates of the three strategies. Also they proposed corresponding optimal power control algorithms for each strategy. The simulation results showed that the proposed algorithms can dramatically improve the transmission rate of cognitive user.

The popular 802.11 MAC protocol provides equal transmission chances to all users, which may achieve throughput-based fairness if all users have the same frame size during a cycle [5]-[8]. Recent studies have shown that time-based fairness is much better than throughput-based fairness in multi-rate WLANs [9].

So far, we have two fairness criteria factors that are widely used in network management: proportional fairness [7] which allocates bandwidth to users in proportion to their bit rates to maximize the sum of the bandwidth utilities of the users, and max-min fairness [10] which allocates throughput as equally as possible through maximizing the minimum throughput. Proportional fairness and time-based fairness are equivalent in multi-rate WLANs when all users have the same weight [11]. The equivalence of max-min fairness and throughput-based fairness under the same condition (integral association) was proved in [12]. 
Qiang Ma, Abdullah Al-Dhelaan and Mznah Al-Rodhaan; Genetic Algorithm based Approach to Enhance Network Performance in Multi-rate WLANs, Transactions on Networks and Communications, Volume 3 No. 6, December (2015); pp: 42-56

The authors in [13] proposed a new algorithm called Power Control for AP (PCAP) to optimize the network utility by maximizing the average and minimizing the variance of the AP utility, the result directly maximized the "throughput" as its target, and then the author started to calculate the " $\mathrm{J}$ " (Jain's fairness index [14]). The author did not mention the "J" at the beginning, though the result showed significant improving of trade-off. We will analyze the relationship between these two variables.

According to IEEE802.11, AP transmission powers can be changed in an allowable range, this technique is called power control. Some previous studies, such as [15]-[16], have assumed that the user-AP associated topology will not change when adjusting the power of APs, so this assumption is not the reality. On the contrary, some papers have noticed this phenomenon and developed techniques called cell breathing [17].

A variable polyhedron genetic algorithm (GA) is proposed in [18], which not only provides an AP service availability guarantee but also yields a near-optimal beacon range for each AP when the number of evolutions is large enough.

The authors in [19] proposed an algorithm that transformed the problem into a monotonic optimization problem. It is solved with geometric programming [20], but it is not suitable for the low Signal to Interference Ratio (SIR) case.

In [21], the authors proposed a centralized algorithm called Non-Linear Approximation Optimization for Proportional Fairness to derive the user-AP association via relaxation, and gave a distributed heuristic called Best Performance First; which provides an AP selection criterion for new comers.

In [22], the authors jointly considered the channel allocation and AP association, aims to maximize the system performance in terms of throughput and fairness. They introduced two penalty functions to relax the constraints, and a discrete particle swarm optimization algorithm to solve the problem.

In [23], to solve the fairness problem in Wireless Mesh Networks (WMNs), the authors proposed a probabilistic approach to provide proportional fairness without solving global non-linear and non-concave optimization. Their Simulation result shows that the proposed solution is better than the standard IEEE 802.11s based EDCA MAC in terms of fairness and throughput.

Similarly in [24], the authors proposed a novel channel assignment algorithm (CAA) to mitigate co-channel interference in Multi-radio Multi-channel (MRMC) wireless mesh networks (WMNs); which is called Topology controlled Interference-aware Channel-assignment Algorithm (TICA). This algorithm uses topology control based on power control to assign channels so that co-channel interference is minimized, network throughput is maximized, and network connectivity is guaranteed. In further, they proposed algorithm using two-way interference-range edge coloring, called Enhanced TICA (e-TICA), which improves the fairness among flows in the network.

In this paper, the contributions are modeling and analysis. The contributions are listed as follows: (a). we describe the "trade-off" using "J of user" and "J of AP", which refer to the fairness of users and fairness of APs respectively, then we study these two variables and derive our simplified coefficient of variation (SCV) model, which is a clear mathematical function to solve such trade-off problem. This is the core contribution of our paper. (b). we define the problem as an informed search NP-hard problem and apply Genetic Algorithm to solve the SCV model. (c). we use multi-channel allocation to improve the 
transmission rate. (d). we use Statistical Analysis System (SAS) for analysis to reveal the relationships of three indicators and the essence of algorithms. (e). SCV opens a door for many Al algorithms; it is a bridge between Network \& Al.

\section{Motivation}

\subsection{The Essence of PCAP: Throughput}

From our SAS analysis in Fig.3, three indicators (Juser: J of user; Jap: J of AP; Tpt: relative Throughput) show that J of AP can represent Throughput (value $>0.8$, so it is highly linear related). Through our Statistics calculation, PCAP focus on J of AP only, which means it only focus on Throughput. This is a deficiency of Target Function design, which is not well reflecting our topic.

\subsection{The Essence of SCV}

The problem is defined as a NP-hard problem since we apply a practical scenario that includes 20 APs, each AP has 10 levels of power, so the state space of the problem will be $10^{20}$, making it neither solvable nor verifiable in polynomial time, which makes it a NP-hard.

From the computation theory, we know that we cannot get an accurate solution. Compared with other NP-hard problems such as TSP (Traveling Salesman Problem), we get some heuristic methods. Since existing models are complicated by using a definition of utility and disturbed by many parameters such as channel gain, those models are not clear enough to apply informed search techniques, so first we need to build a clear, simplified model SCV, and then apply the Genetic Algorithm to solve the model.

Since our topic is: "J of user (fairness of users) \& Throughput", which means to make balance between these two parameters. Obviously the two parameters have different units, then we have to convert the "Throughput" to "J of AP" (already explained, it can represent Throughput, with high linear relation).

Then our SCV offers a new designed target function: $F=(1 /$ Jusers -1$)+\omega(1 /$ Japs -1$)$, which reflects the balance of two parameters (J of user \& Throughput), and we will rewrite to get its final form $f$.

\section{Genetic Algorithm}

The genetic algorithm is an adaptive strategy and a global optimization technique [25]. It is an evolutionary algorithm and belongs to the broader study of evolutionary computation.

The genetic algorithm is inspired by population genetics (including heredity and gene frequencies), and its evolution is at the population level, as well as the understanding of the structure (such as chromosomes, genes, and alleles) and mechanisms (such as recombination and mutation). Individuals of a population contribute their genetic material (called the genotype) in proportion to the suitability of their expressed genome (called their phenotype) to their environment in the form of offspring. The next generation is created through a procedure of mating that involves recombination of two individual genomes in the population with the introduction of random copying errors (called mutations). This iterative procedure may result in an improved adaptive fit between the phenotypes of individuals in a population and the environment.

The goal of the genetic algorithm is to maximize the payoff of candidate solutions in the population against a cost function from the problem domain. The strategy of the genetic algorithm is to repeatedly employ surrogates for the recombination and mutation genetic mechanisms in the population of candidate 
Qiang Ma, Abdullah Al-Dhelaan and Mznah Al-Rodhaan; Genetic Algorithm based Approach to Enhance Network Performance in Multi-rate WLANs, Transactions on Networks and Communications, Volume 3 No. 6, December (2015); pp: 42-56

solutions, where the cost function (also known as objective or fitness function) applied to a decoded representation of a candidate governs the probabilistic contributions a given candidate solution can make to the subsequent generation of candidate solutions.

Simple Genetic Algorithm:

\{

initialize population;

evaluate population;

while Termination Criteria Not Satisfied

\{

select parents for reproduction;

perform recombination and mutation;

evaluate population;

\}

\}

\section{Model Design and Simulation}

Now we are going to explain our SCV model and apply it in Genetic Algorithm.

\subsection{The Way APs Attract Users}

The user will select the strongest received signal strength indicator (RSSI) as default. In the model [26], $R S S I=a P / X^{\alpha}$ where " $a$ " is a constant factor, " $\mathrm{P}$ " is received power, " $\mathrm{X}$ " is distance between user and selected AP, while " $\alpha$ " has different value in different scenarios, generally between 1.6 and 6.5 [27]. The formula only determines the association matrix of User-AP. In practice, the general power range of the AP is $10 \mathrm{dBm} \sim 30 \mathrm{dBm}$, i.e. $1 \mathrm{mw} \sim 1 \mathrm{w}$, here we adopt $\alpha=3$ for indoor case. From the formula, the value of " $a$ " does not affect the association results, to simplify the mathematical form, we take $a=1$, so our model adopts a simplified form:

$$
R S S I=P / X^{3}
$$

\subsection{Study the SINR[ $\left.\mathrm{r}_{\mathrm{ij}}\right]$ of the User[i]}

Assuming the user[i] connects to AP[j], the power of AP[j] is $P_{j}$. Wherein " $g$ " are channel gains, $A_{i}$ is a set of all APs within the same channel of AP[j]. $\mathrm{N}_{0 \mathrm{j}}$ is an additive white Gaussian noise generated by AP[j].

$$
r_{i j}=\frac{g_{i j} p_{j}}{\sum_{k \in A_{i} \cap k \neq j} g_{i k} p_{k}+N_{0 j}}
$$

It is worth noting that $\mathrm{N}_{0 \mathrm{j}}$ can be adjusted to an exact value [28]-[29]. So we can set a constant $\mu>0$,

$$
\mu=\frac{g_{i j}}{\sum_{k \in A_{i} \cap k \neq j} g_{i k} p_{k}+N_{0 j}}
$$




\subsection{Study the Relationship between User[i]'s Transmission Rate $\mathrm{V}_{i}$ and Its SINR [rij]}

Table 1. $v_{i}-r_{i j}$ relationship

\begin{tabular}{|c|c|c|c|c|c|c|c|c|}
\hline$r_{i j}(d B)$ & 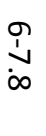 & $\begin{array}{l}v \\
\infty \\
1 \\
\omega\end{array}$ & $\begin{array}{l}0 \\
\stackrel{0}{0} \\
\stackrel{\infty}{0}\end{array}$ & $\begin{array}{l}\mathfrak{0} \\
\dot{0} \\
1 \\
\stackrel{1}{v}\end{array}$ & $\begin{array}{l}\stackrel{\bullet}{N} \\
\stackrel{\infty}{\infty} \\
\infty\end{array}$ & $\begin{array}{l}\stackrel{\infty}{\infty} \\
\infty \\
\sim \\
\sim\end{array}$ & $\begin{array}{l}\tilde{N} \\
\stackrel{\sim}{\dot{N}} \\
\dot{\sigma}\end{array}$ & 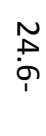 \\
\hline $\mathrm{Vi}_{\mathrm{i}}(\mathrm{Mbps})$ & 6 & 9 & 12 & 18 & 24 & 36 & 48 & 54 \\
\hline
\end{tabular}

From Table 1 in [13], we see the monotonically increasing relationship between the two variables. Here we might assume that two variables meet the linear relationship as an approximation, $v_{i}=\beta r_{i j}, \beta>0$ is a constant of proportionality. Then connect this to (2) and (3) we have: $v_{i}=\beta r_{i j}=\beta \mu p_{j}=\lambda p_{j}$ (4)

$$
\text { So } \lambda \text { is a constant: } \lambda=\beta \mu
$$

\subsection{Study the Effective Speed of $v_{i}$}

Let $N[j]$ denote the total number of users which connect with $A P[j]$. Because the users are time based share the chance of $A P[j]$, so the effective speed of $v_{i}$ is:

$$
\overline{v_{i}}=\frac{\lambda p_{j}}{N[j]}
$$

From this formula we know that it is better to decrease the $\mathrm{N}[\mathrm{j}]$, and increase the $\mathrm{p}_{\mathrm{j}}$ and $\lambda$.

\subsection{Study the AP'S Power}

According to the simulation result in [13], we know that usually 10 levels of AP power will be enough to achieve a good result. Therefore, in our model, $\mathrm{p}_{\max }$ and $\mathrm{p}_{\min }$ have relationship as following:

$p_{\max } / p_{\min }=10, p_{\max }$ will be the basis of calculation, since we need to increase the $p_{j}$, so the 10 power levels are in Table 2.

Table 2. Level-value relationship

\begin{tabular}{|l|c|c|c|c|}
\hline level & 1 & 2 & $\ldots$ & 10 \\
\hline value & $p_{\min }$ & $2 p_{\min }$ & $\ldots$ & $10 p_{\min }$ \\
\hline
\end{tabular}

Note here the unit of power is " $m w$ ", not " $d B m$ ". Since $P_{j}=P_{\min } l_{j}\left(l_{j}=1,2 \ldots 10\right)$, note that $l_{j}$ denotes the level of AP power, so the formula (6) can be rewritten as follows:

$$
\overline{v_{i}}=\frac{\lambda p_{\min } l_{j}}{N[j]}=\lambda p_{\min } \frac{l_{j}}{N[j]}
$$

Let $\mathrm{M}$ be the total number of users and $\mathrm{N}$ be the total number of APs. From statistics we know that the expectation of $\bar{V}_{i}$ for all users is denoted as $E\left(\bar{V}_{i}\right)$, and variance of $\bar{V}_{i}$ for all users is denoted as $S^{2}\left(\bar{V}_{i}\right)$. We have the following $(i=1,2, \ldots M ; j=1,2, \ldots N)$ : 


$$
\begin{gathered}
E\left(\overline{v_{i}}\right)=\lambda p_{\min } E\left(\frac{l_{j}}{N[j]}\right) \\
S^{2}\left(\overline{v_{i}}\right)=\left(\lambda p_{\min }\right)^{2} S^{2}\left(\frac{l_{j}}{N[j]}\right)
\end{gathered}
$$

Let $b[i]$ denote the average transmission speed from user[i] to AP[j], we have $b[i]=\bar{V}_{i}$. Moreover, let $U[j]$ denote the transmission speed from the AP[j] to backbone. The Expectation of $b[i]$ is denoted as: $E(b[i])$, and Variance of $\mathrm{b}[\mathrm{i}]$ is denoted as: $S^{2}(b[i])$, and Expectation of $U[\mathrm{j}]$ is denoted as: $E(U[j])$, and Variance of $U[j]$ is denoted as: $S^{2}(U[j])$, so continue we have formulas as following:

$$
\begin{gathered}
E(b[i])=E\left(\overline{v_{i}}\right)=\lambda p_{\min } E\left(\frac{l_{j}}{N[j]}\right) \\
S^{2}(b[i])=S^{2}\left(\overline{v_{i}}\right)=\left(\lambda p_{\min }\right)^{2} S^{2}\left(\frac{l_{j}}{N[j]}\right) \\
E(U[j])=E\left(\sum_{u \in[i] \rightarrow A P[j]} b[i]\right)=E\left(\lambda p_{\min } N[j] \frac{l_{j}}{N[j]}\right)=\lambda p_{\min } E\left(l_{j}\right) \\
S^{2}(U[j])=S^{2}\left(\sum_{\operatorname{Leg}[i] \rightarrow A P[j]} b[i]=S^{2}\left(\lambda p_{\min } N[j] \frac{l_{j}}{M j j]}\right)=\left(\lambda p_{\min }\right)^{2} S^{2}\left(l_{j}\right)\right.
\end{gathered}
$$

Let cvusers denote the coefficient of variation of transmission speed of all users and cvAPs denote the coefficient of variation of transmission speed of all APs, we have:

$$
\begin{gathered}
\text { cvusers }^{2}=\frac{S^{2}(b[i])}{E^{2}(b[i])}=\frac{\left(\lambda p_{\min }\right)^{2} S^{2}\left(\frac{l_{j}}{N[j]}\right)}{\left(\lambda p_{\min }\right)^{2} E^{2}\left(\frac{l_{j}}{N[j]}\right)}=\frac{S^{2}\left(\frac{l_{j}}{N[j]}\right)}{E^{2}\left(\frac{l_{j}}{N[j]}\right)}=\frac{M \sum_{i=1, b[i] \rightarrow A P[j]}^{M} \frac{l_{j}^{2}}{N^{2}[j]}}{\left(\sum_{j=1}^{N} l_{j}\right)^{2}}-1 \\
=\frac{M \sum_{j=1}^{N} \frac{l_{j}^{2}}{N[j]}}{\left(\sum_{j=1}^{N} l_{j}\right)^{2}}-1=\frac{1}{J_{u s e r s}}-1 \\
\operatorname{cvAPS}^{2}=\frac{S^{2}(U[j])}{E^{2}(U[j])}=\frac{\left(\lambda p_{\min }\right)^{2} S^{2}\left(l_{j}\right)}{\left(\lambda p_{\min }\right)^{2} E^{2}\left(l_{j}\right)}=\frac{S^{2}\left(l_{j}\right)}{E^{2}\left(l_{j}\right)}=\frac{N \sum_{j=1}^{N} l_{j}^{2}}{\left(\sum_{j=1}^{N} l_{j}\right)^{2}}-1=\frac{1}{J_{A P s}}-1
\end{gathered}
$$

Note, here we adopt the definition of J in [13], where we have $J_{x}=\left(\sum_{i=1}^{n} x_{i}\right)^{2} / n \sum_{i=1}^{n} x_{i}^{2}$, above is the relationship between J and the square of coefficient of variation. 


\subsection{Cost Function $f$ Construction}

According to our topic, we need a function that can describe the tradeoff between fairness of users and throughput of network. In [13], the algorithm is divided into two steps: increase average value and decrease variance value of AP utility to increase throughput of network. They are equal to decreasing $c v A P s$ or $c v A P s^{2}$. So increasing $\mathrm{J}$ of users is equal to decreasing cvusers ${ }^{2}$.

Let $\mathrm{F}$ denote a target function as follows: $F=$ cvusers $^{2}+\omega\left(c v A P s^{2}\right), \omega$ is weight proportion factor, it is very important reflecting our requirement how to make the balance between fairness and throughput, it is a quantifiable indicator.

Here we do some mathematical derivation to illustrate how we get a reasonable value of $\omega$. Considering the static grouping problem: $m$ numbers are average divided by $n$ groups, therefore each group has $m / n$ numbers. Given that the expectation of total numbers is $\sigma$, and their variance is $s^{2}$, so for group[i] we have:

$$
\begin{aligned}
E(\operatorname{group}[i])= & E\left(\sum_{\text {number }[j] \in \text { group }[i]} \text { number }[j]\right)=\sum_{\text {number }[j] \in \text { group }[i]} E(\text { number }[j])=\frac{m}{n} \sigma \\
S^{2}(\operatorname{group}[i])= & S^{2}\left(\sum_{\text {number }[j] \in \text { group }[i]} \text { number }[j]\right)=\sum_{\text {number }[j] \in \text { group }[i]} S^{2}(\text { number }[j])=\frac{m}{n} s^{2} \\
\text { vvnumbers }^{2}= & \frac{s^{2}}{\sigma^{2}} \\
& \operatorname{cvgroups}^{2}=\frac{S^{2}(\operatorname{group}[i])}{E^{2}(\operatorname{group}[i])}=\frac{\frac{m}{n} s^{2}}{\left(\frac{m}{n}\right)^{2} \sigma^{2}}=\frac{1}{\frac{m}{n}} \frac{s^{2}}{\sigma^{2}}=\frac{1}{\frac{m}{n}} \text { cvnumbers }^{2}
\end{aligned}
$$

So it means cvgroups $^{2}$ is much smaller than cvnumbers $^{2}$, comparing this example to our function " $F$ ", in function " $F$ " we should amplify the small part since two parts have relationship. So we decide to give value to $\omega$, let $\omega=M / N$.

$$
F=\text { cvusers }^{2}+\omega\left(\operatorname{cvAPs}^{2}\right)=\frac{M \sum_{j=1}^{N} \frac{l_{j}^{2}}{N[j]}}{\left(\sum_{j=1}^{N} l_{j}\right)^{2}}-1+\frac{M}{N}\left(\frac{N \sum_{j=1}^{N} l_{j}^{2}}{\left(\sum_{j=1}^{N} l_{j}\right)^{2}}-1\right)=M \frac{\left(\sum_{j=1}^{N} \frac{l_{j}^{2}}{N[j]}+\sum_{j=1}^{N} l_{j}^{2}\right)}{\left(\sum_{j=1}^{N} l_{j}\right)^{2}}-\left(1+\frac{M}{N}\right)=M f-\left(1+\frac{M}{N}\right)
$$

Wherein:

$$
f=\frac{\left(\sum_{j=1}^{N} \frac{l_{j}^{2}}{N[j]}+\sum_{j=1}^{N} l_{j}^{2}\right)}{\left(\sum_{j=1}^{N} l_{j}\right)^{2}}=\frac{\sum_{j=1}^{N}\left(l_{j}^{2}\left(1+\frac{1}{N[j]}\right)\right)}{\left(\sum_{j=1}^{N} l_{j}\right)^{2}}
$$

Note that $\mathrm{M}$ and $\mathrm{N}$ are constants as defined before. $\mathrm{M}$ is total number of users, $\mathrm{N}$ is total number of APs. When " $F$ " goes to minimum, it is equal to " $f$ " goes to minimum. So (18) will be our simplified target function, to achieve the purpose of the tradeoff between Fairness (users) and Throughput (network). 


\subsection{Throughput}

From formula (12) we know that:

$$
\begin{gathered}
\text { Throughput }_{\text {real }}=\sum_{j=1}^{N} U[j]=\lambda p_{\min } \sum_{j=1}^{N} l_{j}=\lambda p_{\min } \text { Throughput }_{\text {relative }} \\
\text { Throughput }_{\text {relative }}=\sum_{j=1}^{N} l_{j}
\end{gathered}
$$

Since the $\lambda p_{\min }$ is constant, we use Throughput ${ }_{\text {relative }}$ to represent Throughput real $_{\text {. }}$.

\subsection{Genetic Algorithm Design and Simulation}

In this part we are going to place a total number of N=20 APs on a 4 by 5 grid, with each AP on a grid point. The coverage area of each AP can across the whole area. The distance between two adjacent APs is set to 100 meters. The maximum transmit power of each AP is set to $20 \mathrm{dBm}(100 \mathrm{mw})$, and so according to our model, the minimum transmission power of each AP is set to $100 / 10=10 \mathrm{mw}=10 \mathrm{dBm}$.

We arrange $M=200$ users random distributed in the whole area. According to [30], a separation of four channels can be used without reducing the performance, so the possibilities could be opened to channels 1, 5, 9 and 13. In this paper we decide to use these channels in order to get a bigger Throughput real .

Let $A P_{j} \rightarrow$ Ci denote $A P_{j}$ using channel $i$, we use $1,5,9,13$ these channels to configure the network as in Table 3.

Table 3. AP-Channel relationship

\begin{tabular}{|l|l|l|l|l|}
\hline$A P_{1} \rightarrow C 1$ & $A P_{2} \rightarrow C 9$ & $A P_{3} \rightarrow C 1$ & $A P_{4} \rightarrow C 9$ & $A P_{5} \rightarrow C 1$ \\
\hline$A P_{6} \rightarrow C 5$ & $A P_{7} \rightarrow C 13$ & $A P_{8} \rightarrow C 5$ & $A P_{9} \rightarrow C 13$ & $A P_{10} \rightarrow C 5$ \\
\hline$A P_{11} \rightarrow C 9$ & $A P_{12} \rightarrow C 1$ & $A P_{13} \rightarrow C 9$ & $A P_{14} \rightarrow C 1$ & $A P_{15} \rightarrow C 9$ \\
\hline$A P_{16} \rightarrow C 13$ & $A P_{17} \rightarrow C 5$ & $A P_{18} \rightarrow C 13$ & $A P_{19} \rightarrow C 5$ & $A P_{20} \rightarrow C 13$ \\
\hline
\end{tabular}

a. Chromosome Coding: the whole path from $A P_{1}$ to $A P_{20}$ is denoted as a Chromosome, and each element is denoted as a Gene, the value of Gene is the level of AP's power. In Table 4 . Where $g e_{i j}=1,2, \ldots 10$

Table 4. Chromosome Coding

\begin{tabular}{|c|c|c|c|c|c|}
\hline Chro\Gene & 1 & $\ldots$ & $\mathrm{j}$ & $\ldots$ & 20 \\
\hline Chro $_{i}$ & $g e_{i 1}$ & $\ldots$ & $g e_{i j}$ & $\ldots$ & $g e_{i 20}$ \\
\hline
\end{tabular}

b. Fitness Function is $f$ in (18), since all parameters' values in algorithm can affect the result of the calculation, the following parameters' values are better after repeated tests.

$\mathrm{C}=50$; denotes the total number of generations.

$\mathrm{Ps}=0.6$; denotes the probability of selection operation.

$\mathrm{Pc}=0.9$; denotes the probability of crossover operation.

$\mathrm{Pm}=0.1$; denotes the probability of mutation operation. 


\section{Result Analysis}

The solution of Genetic Algorithm has many parameters. Since all parameters' values in algorithm can affect the result of the calculation, we need to run multiple times on the same data and same parameters, and select the best, average, or representative results, also search for the more suitable parameters'

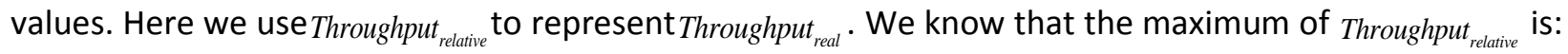
$10 \times 20=200$, but it will never be achieved, at least because of users' distribution.

The simulation results show in the following figures. Generally, in genetic algorithm, when the best individuals of each generation are saved then the simulation result will converge. According to fitness function, we save enough best chromosomes and phase out those worst ones, therefore, each parameter is convergent, since random factors exist in algorithm, so there is slight vibration around the horizontal line.

From the Figure $1 \&$ Figure 2 we can see there are totally 52 generations in the experiment. We select the best path in each generation and calculate the values of those indicators in this path, then plot these values for all 52 generations. In Fig.1, max J of user denotes the maximum fairness value of users in that generation, max J of AP denotes the maximum fairness value of APs in that generation. In Figure 2, max Throughput of network denotes the maximum value of Throughput relative $_{\text {in }}$ that generation, min cost of $f$ denotes the minimum value of the function in (18).

From the Fig.1, at the $21^{\text {st }}$ generation, the biggest $\mathrm{J}$ of user is almost equal to 0.84 , and corresponding $\mathrm{J}$ of AP is almost equal to 0.99 , while in the Fig.2, at the $21^{\text {st }}$ generation the Throughput relative $_{\text {is }}$ is almost equal to 169, since its maximum value is 200 , then the throughput of the network is almost equal to $169 / 200 \approx 85 \%$ of the network bandwidth. Moreover the cost of $f$ in $21^{\text {st }}$ generation is 0.057 . So from the figures, the administrator may choose this generation to configure the network.
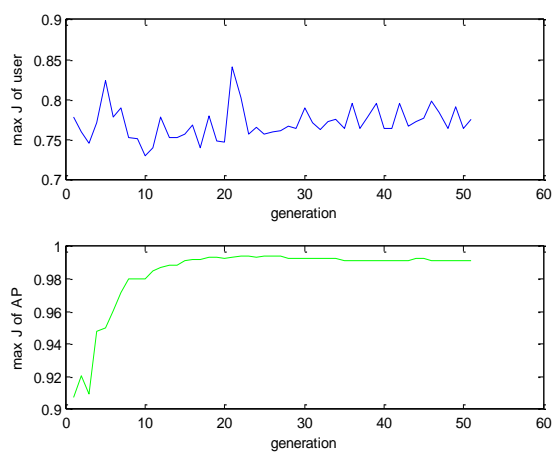

Figure 1. $\{\max \mathrm{J}$ of user, max J of AP\}-generation plot
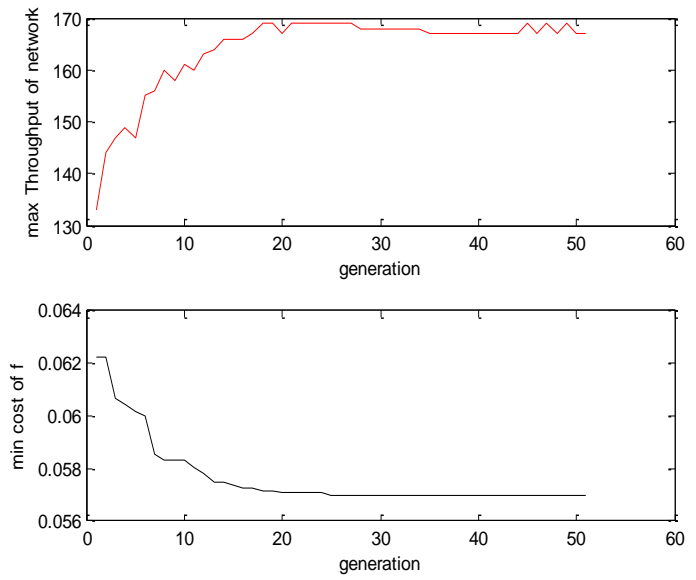

Figure 2. \{max Throughput of network, min cost of $\mathrm{f}$-generation plot

SAS Analysis: we use the samples from experimental data to study the correlation coefficients among these indicators. Wherein Juser denotes J of user, Jap denotes J of AP, Tpt denotes Throughput relative, cost denotes the value of function $f$ in (18). 


\begin{tabular}{|c|c|c|c|c|}
\hline \multicolumn{5}{|c|}{$\begin{array}{c}\text { Pearson Correlation Coefficients, } N=326 \\
\text { Prob }>|r| \text { under } \mathrm{HO}: \text { Rho }=0\end{array}$} \\
\hline & cost & Tpt & Jap & Juser \\
\hline $\begin{array}{l}\text { cost } \\
\text { cost }\end{array}$ & 1.00000 & $\begin{array}{r}-0.80950 \\
<.0001\end{array}$ & $\begin{array}{r}-0.94339 \\
<.0001\end{array}$ & $\begin{array}{r}-0.31595 \\
<.0001\end{array}$ \\
\hline $\begin{array}{l}\text { Tpt } \\
\text { Tpt }\end{array}$ & $\begin{array}{r}-0.80950 \\
<.0001\end{array}$ & 1.00000 & $\begin{array}{r}0.80200 \\
<.0001\end{array}$ & $\begin{array}{r}0.17151 \\
0.0019\end{array}$ \\
\hline $\begin{array}{l}\text { Jap } \\
\text { Jap }\end{array}$ & $\begin{array}{r}-0.94339 \\
<.0001\end{array}$ & $\begin{array}{r}0.80200 \\
<.0001\end{array}$ & 1.00000 & $\begin{array}{r}0.11425 \\
0.0392\end{array}$ \\
\hline $\begin{array}{l}\text { Juser } \\
\text { Juser }\end{array}$ & $\begin{array}{r}-0.31595 \\
<.0001\end{array}$ & $\begin{array}{r}0.17151 \\
0.0019\end{array}$ & $\begin{array}{r}0.11425 \\
0.0392\end{array}$ & 1.00000 \\
\hline
\end{tabular}

Figure 3. correlation coefficients.

Figure 3 shows that at alpha= 0.05 significance level, all the $p$-values are less than 0.05 , so we reject the $\mathrm{H}_{0}$, and accept $\mathrm{H}_{1}$ that these variables are linearly related, wherein the Tpt-(Jap, Juser) have highly significant linear correlations, while correlations of Jap-Juser is weak. We compared the degree of concentration of those data points in Fig. 4 and Fig.5. It is clear that data points are more concentrated in Fig.5. This means the linear correlation of Tpt-Jap is much higher than the linear correlation of Tpt-Juser, which also proves the effectiveness of SCV model (coefficient of Tpt-Jap >0.8, Tpt-Juser $=0.17$, so it is more effective to use J of AP whereas not J of user to represent the Throughput).

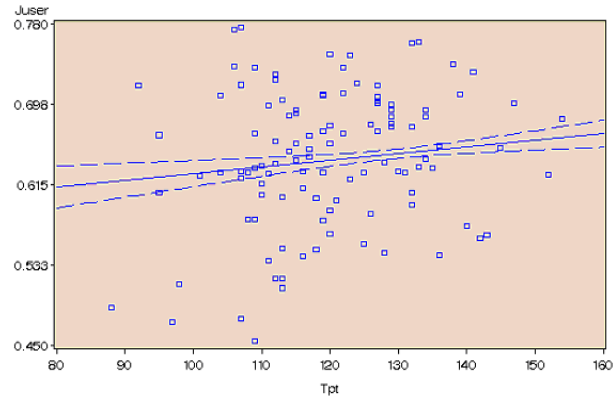

Figure 4. Tpt-Juser linear regressions

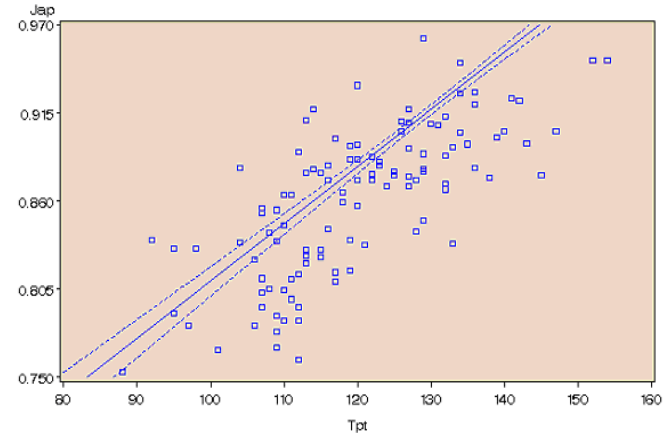

Figure 5. Tpt-Jap linear regression

\section{Comparison Analysis:}

Table 5. The Statistics of the Results

\begin{tabular}{|c|c|c|c|c|c|}
\hline Algorithm & $\begin{array}{c}\text { Average } \mathrm{AP} \\
\text { Utility }\left(U^{a}\right)\end{array}$ & $\begin{array}{c}\text { AP Utility } \\
\text { Variance } \\
\left(\sigma_{u}\right)\end{array}$ & $\begin{array}{c}\text { Total } \\
\text { Network } \\
\text { Utility }\end{array}$ & $\begin{array}{c}\text { Jain's } \\
\text { Fairness } \\
\text { Index }\end{array}$ & $\begin{array}{c}\text { Average } \\
\text { Power } \\
(\mathrm{dBm})\end{array}$ \\
\hline PCAP & $3.82 \times 10^{9}$ & $2.98 \times 10^{9}$ & 117.42 & 0.90 & 17.15 \\
MARL & $2.67 \times 10^{9}$ & $5.00 \times 10^{9}$ & 102.30 & 0.85 & 17.28 \\
SR & $1.46 \times 10^{9}$ & $12.80 \times 10^{9}$ & 99.66 & 0.75 & 18.06 \\
SSF & $1.11 \times 10^{9}$ & $27.31 \times 10^{9}$ & 79.59 & 0.37 & 20.00 \\
\hline
\end{tabular}

Here we want to compare our solution with PCAP in [13], we can see the above Table 5 from [13],

since we use different definitions to denote throughput of AP and throughput of network, we have to use the indirect method to illustrate some issues.

According to [13], we can transfer and calculate their J of AP:

$$
c v A P s^{2}=\frac{S^{2}(U[j])}{E^{2}(U[j])}=\frac{\log \left(2.98 \times 10^{9}\right)}{\left(\log \left(3.82 \times 10^{9}\right)\right)^{2}}=0.1=\frac{1}{J_{A P s}}-1
$$




$$
\text { So their } J_{A P_{s}}=0.9=J_{\text {users }}
$$

And we have: $U \leq n \log \left(\overline{U^{a}}\right)=20 \log \left(3.82 \times 10^{9}\right)=191.64=U_{\max }$

Then their throughput percentage of network bandwidth is:

$$
U_{\text {network:utility }} / U_{\max }=117.42 / 191.64 \approx 61.3 \%
$$

In [13], the authors set 300 users and 16 APs (the other 4 APs actually became useless under their assumption).

We select average case in Fig.1, at the $30^{\text {th }}$ generation, the $\mathrm{J}$ of user is almost equal to 0.79 , and corresponding J of AP is almost equal to 0.99 , the Throughput ${ }_{\text {relative }}$ is almost equal to 168 , since its maximum value is 200 as mentioned before, then the throughput of the network is equal to $168 / 200=84 \%$ of the network bandwidth. And the corresponding cost of $f$ is almost equal to 0.057 .

In Figure 1, our J of AP is superior to theirs in (22). From the throughput point of view, our throughput percentage of network bandwidth is $84 \%>61.3 \%$ in (24), so our method is better than PCAP. But from the fairness of users(J of user) point of view, PCAP is better than ours since $0.79<0.9$ in (22).

According to (17), we convert (22) into our function F, we have:

$$
\begin{gathered}
F_{P C A P}=\left[\left(1 / J_{\text {users }}\right)-1\right]+(M / N)\left[\left(1 / J_{A P_{s}}\right)-1\right]=[(1 / 0.9)-1]+(300 / 16)[(1 / 0.9)-1]=2.17 \\
F_{S C V}=M f-(1+M / N)=200 \times 0.057-(1+200 / 20)=0.4
\end{gathered}
$$

So the overall performance depends on the requirement of administrators, what indicator they most concern. Here we define the value of " $\mathrm{F}$ " as the overall performance criteria of algorithm, note smaller "F" is better then from (25) and (26) we know that our SCV model is much better than PCAP. The above comparison analysis result is in Table 6.

Table 6. Comparison Result

\begin{tabular}{|l|c|c|}
\hline & PCAP & SCV-gen \\
\hline J of user ( win) & 0.9 & 0.79 \\
\hline J of AP ( win) & 0.9 & 0.99 \\
\hline Throughput \% ( $\uparrow$ win) & $61.3 \%$ & $84 \%$ \\
\hline Function “F” value ( $\downarrow$ win) & 2.17 & 0.4 \\
\hline
\end{tabular}

Theoretically, our design of target function " $F$ " in (17) is more simple and rational than PCAP algorithm, since we joint consider the J of user and Throughput (represented by J of AP), we regard them as two variables to reflect our topic. While the target of PCAP is the Throughput, the author used two subalgorithms to achieve J of AP only, and then got their by-product: J of user.

Technically, our SCV math model is a door that leads this problem to Al algorithms. The clear target function " $\mathrm{F}$ " is easy to be applied to other Al algorithms, while PCAP cannot

\section{Conclusions}

The objective of this paper is to improve the trade-off between user fairness ( $\mathrm{J}$ of user) and network throughput(represented by J of AP) via power control in multi-rate WLANs. 
Qiang Ma, Abdullah Al-Dhelaan and Mznah Al-Rodhaan; Genetic Algorithm based Approach to Enhance Network Performance in Multi-rate WLANs, Transactions on Networks and Communications, Volume 3 No. 6, December (2015); pp: 42-56

In this article, we first construct a new simplified model called SCV. The goal of the model is to derive a target function " $F$ " (17) and its simplified form " $f$ " (18) as our key foundation. Then we use Genetic Algorithm to solve our model, we conduct a simulation in Matlab. After that we give analysis of our SCV model and simulation results which confirm that our model is efficient and superior to PCAP in some aspects and overall performance under a new criteria of algorithm designed for such specific problem. In addition, based on the data samples from the state space, we use SAS to conduct correlationship analysis mainly among three indicators, and reveal their relationships.

SCV opens a door for many Al algorithms to apply in this problem, it is a bridge between Network \& Al.

Our future work is to derive a more accurate target function, and adjust the values of parameters to find more suitable combination so that to improve the results. Also we are working on other Al solutions based on SCV model.

\section{ACKNOWLEDGMENT}

The authors extend their appreciation to the Deanship of Scientific Research at King Saud University for funding this work through research group no. RGP-264.

\section{REFERENCES}

[1]. Qiang Ma, Abdullah Al-Dhelaan, Mznah Al-Rodhaan, "Using Genetic Algorithm to Improve Tradeoff between Fairness and Throughput in Multi-Rate WLANs", Proc. 8th WSEAS International Conference on Circuits, Systems and Signals (CSS'15), Michigan State University, USA, September 2015.

[2]. Jain-Shing Liu, "AchievingWeighted Fairness in IEEE 802.11-based WLANs: Models and Analysis", WSEAS Transactions on Communications, Issue 6, vol. 7, June 2008.

[3]. Yaser Mahmood, A. Hamid, D. K. Lobiyal. "Improved Power Control MAC Protocol for wireless Ad Hoc Networks", WSEAS Transactions on Communications, Issue 1, vol. 10, January 2011.

[4]. Qian Hu, Zhenzhou Tang, "Study on Power and Rate Control Algorithm for Cognitive Wireless Networks", WSEAS Transactions on Communications, Issue 4, vol. 9, April 2010.

[5]. G. Tan, J. Guttag, "Time-based fairness improves performance in multi-rate WLANs", Proc. Usenix Annual Technical Conf., 2004, pp. 23-23.

[6]. M. Heusse, F. Rousseau, G. Berger Sabbatel, A. Duda, "Performance anomaly of 802.11 b", Proc. IEEE INFOCOM, 2003, pp. 836-843.

[7]. F. P. Kelly, "Charging and rate control for elastic traffic", European Trans. Telecommun., vol. 8, no. 1, 1997. 
[8]. A. Banchs, P. Serrano, H. Oliver, "Proportional fair throughput allocation in multi-rate IEEE 802.11e wireless LANs", Wireless Networks, vol. 13, no. 5, pp. 649-662, 2007.

[9]. A. V. Babu, L. Jacob, "Performance analysis of IEEE 802.11 multi-rate WLANs: time based fairness vs throughput based fairness", Proc. IEEE Int. Conf. on Wireless Networks, Comm. and Mobile Compu., pp. 203-208. 2005.

[10]. D. Bertsekas, R. Gallager, “Data Networks”, Prentice-Hall, 1987.

[11]. W. Li, Y. Cui, S. Wang, X. Cheng, "Approximate optimization for proportional fair AP association in multi-rate WLANs", Proc. 5th Int. Conf. WASA, 2010, pp. 36-46.

[12]. Y. Bejerano, S. J. Han, L. E. Li, "Fairness and load balancing in wireless LANs using association control", IEEE/ACM Trans. Network., vol. 15, no. 3, pp. 560-573, 2007.

[13]. Wei Li, Yong Cui, Xiuzhen Cheng, Mznah A. Al Rodhaan, Abdullah Al Dhelaan, "Achieving Proportional Fairness via AP Power Control in Multi-Rate WLANs", IEEE Transactions on Wireless Comm., vol. 10, no. 11, November 2011.

[14]. R. Jain, D. M. Chiu, W. R. Hawe, "A quantitative measure of fairness and discrimination for resource allocation in shared computer system", Digital Equipment, Tech. Dec-Tr-301, 1984.

[15]. V. P. Mhatre, K. Papagiannaki, F. Baccelli, "Interference mitigation through power control in high density 802.11 WLANs", Proc. IEEE INFOCOM, pp. 535-543, 2007.

[16]. V. Hasu, V. Koivo, "Fair transmission rate allocation: a power control feasibility approach", Proc. IEEE ICCS, pp. 1-5, 2006.

[17]. Y. Bejerano, S. J. Han, "Cell breathing techniques for load balancing in wireless LANs", IEEE Trans. Mobile Computing, vol. 8, no. 6, 2009.

[18]. Shengling Wang, Jianhui Huang, Xiuzhen Cheng, Biao Chen, "Coverage adjustment for load balancing with an AP service availability guarantee in WLANs", Wireless Networks, April 2014, vol 20, Issue 3, pp. 475-491.

[19]. L. P. Qian, Y. Jun, "Monotonic optimization for non-concave power control in multiuser multicarrier network systems", Proc. IEEE INFOCOM, 2009, pp. 172-180.

[20]. M. Chiang, C. W. Tan, D. P. Palomar, D. O'Neill, D. Julian, "Power control by geometric programming”, IEEE Trans. Wireless Commun., vol. 6, no. 7, pp. 2640-2651, 2007.

[21]. Wei Li, Shengling Wang, Yong Cui, Xiuzhen Cheng, Ran Xin, Mznah A. Al-Rodhaan, Abdullah AlDhelaan, "AP Association for Proportional Fairness in Multirate WLANs", IEEE/ACM Trans. on Networking, vol. 22, no. 1, February 2014. 
Qiang Ma, Abdullah Al-Dhelaan and Mznah Al-Rodhaan; Genetic Algorithm based Approach to Enhance Network Performance in Multi-rate WLANs, Transactions on Networks and Communications, Volume 3 No. 6, December (2015); pp: 42-56

[22]. Xiaohui Chen, Wenqing Cheng, Wei Yuan, Wei Liu, Jing Xu, "Joint Optimization of Channel Allocation and AP Association in Variable Channel-width WLANs", 2013 IEEE Wireless Communications and Networking Conference: MAC.

[23]. Sandip Chakraborty, Pravati Swain, Sukumar Nandi, "Proportional fairness in MAC layer channel access of IEEE 802.11s EDCA based wireless mesh networks", Ad Hoc Networks, 2013, vol.11 (1).

[24]. Aizaz U Chaudhry, Nazia Ahmad, Roshdy HM Hafez, "Improving throughput and fairness by improved channel assignment using topology control based on power control for multi-radio multi-channel wireless mesh networks", EURASIP Journal on Wireless Comm. and Networking, 2012, vol.2012 (1), pp.1-25.

[25]. Jason Brownlee, “Clever Algorithms: Nature Inspired Programming Recipes”, LuLu, first edition, January 2011, ISBN: 978-1-4467-8506.

[26]. DING Xiaole, LI Fenghua, LI Hewu, JIANG Yong, WU Jianping, "Dynamic load balancing mechanism in WLAN Based on Power Control and Location Information", Journal of Xiamen University(Natural Science), vol.46, Sup 2, Nov. 2007.

[27]. Andrea Goldsmith, "Wireless Communications", Stanford University, 2004.

[28]. Yin Zhongqiu, Shi Chunhe, Chen Mingsheng, Liu Shuzhong, "A White and Gaussian White Noise Generator with Adjustable Parameters", Fire Control and Command Control, 2008.08.

[29]. Wang Pengyu, Zhai Lili, Shi Jufeng, "Design of Gaussian White Noise Generator with Adjustable Parameters Based on FPGA", Shipboard Electronic Countermeasure, doi: 10.3969/j.issn.16739167.2013.04.029

[30]. Eduard Garcia Villegas, Elena López-Aguilera, Rafael Vidal, Josep Paradells, "Effect of adjacentchannel interference in IEEE 802.11 WLANs", Cognitive Radio Oriented Wireless Networks and Communications, 2007. 2nd International Conference, doi:10.1109/crowncom.2007.4549783. 